\title{
AN ISOMETRY THEOREM FOR QUADRATIC DIFFERENTIALS ON RIEMANN SURFACES OF FINITE GENUS
}

\author{
NIKOLA LAKIC
}

\begin{abstract}
Assume both $X$ and $Y$ are Riemann surfaces which are subsets of compact Riemann surfaces $X_{1}$ and $Y_{1}$, respectively, and that the set $X_{1}-X$ has infinitely many points. We show that the only surjective complex linear isometries between the spaces of integrable holomorphic quadratic differentials on $X$ and $Y$ are the ones induced by conformal homeomorphisms and complex constants of modulus 1 . It follows that every biholomorphic map from the Teichmüller space of $X$ onto the Teichmüller space of $Y$ is induced by some quasiconformal map of $X$ onto $Y$. Consequently we can find an uncountable set of Riemann surfaces whose Teichmüller spaces are not biholomorphically equivalent.
\end{abstract}

\section{INTRODUCTION}

Let $A(X)$ and $A(Y)$ be the complex Banach spaces of integrable holomorphic quadratic differentials on the Riemann surfaces $X$ and $Y$. If $\alpha$ is a conformal mapping from $Y$ onto $X$, and $C$ is a complex constant of modulus one, then $C \alpha^{*}$ : $A(X) \rightarrow A(Y)$ defined by $C \alpha^{*}(\varphi)(z)=C \varphi(\alpha(z)) \alpha^{\prime}(z)^{2}$ is a complex linear isometry in the norms for $A(X)$ and $A(Y)$.

Our main result is the following theorem.

Isometry Theorem. If the Riemann surfaces $X$ and $Y$ are subsets of compact Riemann surfaces $X_{1}$ and $Y_{1}$, respectively, and the set $X_{1}-X$ has infinitely many points, then every linear isometry $L$ from $A(X)$ onto $A(Y)$ comes from pull-back by a conformal mapping $\alpha$ from $Y$ onto $X$ and multiplication by a complex constant $C$ of modulus one, i.e. $L=C \alpha^{*}$.

A Riemann surface is of finite analytic type $(g, n)$ if it is obtained from a compact Riemann surface of genus $g$ by deleting $n$ points. A Riemann surface of finite analytic type $(g, n)$ is called exceptional if $2 g+n \leq 4$. For the case of Riemann surfaces of type $(g, 0), g \geq 2$, the isometry theorem was proved by Royden ( $[\mathrm{R}])$. Using the same method of proof, Earle and Kra generalized the isometry theorem to the case of nonexceptional Riemann surfaces of finite analytic type. The method was to study the smoothness properties of the $L^{1}$-norm on $A(X)$ and $A(Y)$ and to consider the differentials in $A(X)$ and $A(Y)$ with zeros of the highest possible order at the points in $X$ and $Y$. This method does not generalize to the case where $X$ is not of finite analytic type because there is no limit on the order of zero of a differential in $A(X)$.

Received by the editors December 1, 1994 and, in revised form, February 26, 1996.

1991 Mathematics Subject Classification. Primary 32G15; Secondary 30C62, 30C75.

(C)1997 American Mathematical Society 
A Riemann surface is of finite genus if it can be holomorphically imbedded into a subset of a compact Riemann surface. We prove the isometry theorem in the case where $X$ and $Y$ are subsets of compact Riemann surfaces $X_{1}$ and $Y_{1}$ such that the set $X_{1}-X$ has infinitely many points. Therefore $X$ and $Y$ are Riemann surfaces of finite genus. The further condition imposed on $X$ simply means that $X$ is not a Riemann surface of finite analytic type, a case completely discussed in [EK]. Hence, we prove the isometry theorem precisely in the case when $X$ is a Riemann surface of finite genus and infinite analytic type and $Y$ is a Riemann surface of finite genus. In the proof we use the smoothness of the $L^{1}$-norm on $A(X)$ and $A(Y)$, and we consider differentials in $A(X)$ with at least a double zero at some fixed point in $X$.

In Section 1 it is shown that the integrability of the quadratic differential $\psi^{2} / \varphi$, where $\psi$ and $\varphi$ are in $A(X)$, is an invariant for every linear isometry $L$ from $A(X)$ onto $A(Y)$. That is obtained by proving that the integrability of $\psi^{2} / \varphi$ is equivalent to the existence of the second derivative of the function $f(t)=\|\varphi+t \psi\|$ at $t=0$, in the direction of both real and imaginary axes.

In Section 2 we consider the case where $X$ and $Y$ are plane domains. We show that the image under $L$ of the space of all quadratic differentials in $A(X)$ with at least a double zero at some fixed point $p$ in $X$ is the space of all quadratic differentials in $A(Y)$ with at least a double zero at some point $q$ in $Y$. That implies that the image under $L$ of the space of all differentials in $A(X)$ that vanish at $p$ is the space of all differentials in $A(Y)$ that vanish at $q$. The function from $Y$ to $X$ that sends $q$ to $p$ is a conformal homeomorphism which together with a complex constant of modulus 1 realizes the given isometry.

We prove the isometry theorem in Section 3, following the same steps from the plane domain case and using standard theorems about the existence of certain meromorphic functions on compact Riemann surfaces.

Finally, in Section 4 we use the isometry theorem to classify the biholomorphic mappings between the Teichmüller spaces of $X$ and $Y$. By combining the isometry theorem with the results in $[\mathrm{EG}]$ we obtain the

Automorphism Theorem. If the Riemann surfaces $X$ and $Y$ are subsets of compact Riemann surfaces $X_{1}$ and $Y_{1}$, respectively, and the set $X_{1}-X$ has infinitely many points, then every holomorphic isomorphism from Teich $(X)$ onto Teich $(Y)$ is induced by a quasiconformal homeomorphism $g$ from $X$ onto $Y$. In particular, every biholomorphic self-mapping of Teich $(X)$ is induced by a quasiconformal self-mapping of $X$.

As an application of the automorphism theorem we prove the existence of uncountably many non-isomorphic Teichmüller spaces by constructing an uncountable set of quasiconformally inequivalent plane regions.

I would like to thank my adviser Frederick P. Gardiner for motivation and many helpful discussions and suggestions. Clifford J. Earle and the referee also made several important suggestions. The remark in Section 2 was pointed out to me by the referee.

\section{The nONSmoothness of ThE NORM ON QUADRATIC DIFFERENTIALS}

Definition 1. If $X$ is a Riemann surface, then $A(X)$ is the Banach space of all holomorphic quadratic differentials $\varphi$ on $X$ satisfying $\|\varphi\|=\iint_{X}|\varphi|<\infty$. 
Note that if $\varphi$ and $\psi$ are two differentials in $A(X)$, then $\frac{\psi^{2}}{\varphi}$ is also a quadratic differential on $X$.

In this section we use the smoothness properties of Teichmüller's metric to show that the integrability of a quadratic differential $\frac{\psi^{2}}{\varphi}$ is an invariant for any complex linear isometry $L$ from $A(X)$ onto $A(Y)$. First we state the following simple inequality for complex numbers and reproduce its short proof from $[\mathrm{H}]$.

Lemma 1. For all complex numbers $z \neq 1, \quad\left|\frac{1-z}{|1-z|}-1\right| \leq 2|z|$.

Proof. Let $1-z=w$. Then

$$
\left|\frac{w}{|w|}-1\right| \leq\left|\frac{w}{|w|}-w\right|+|w-1|=|1-| w||+|w-1| \leq 2|w-1| .
$$

Lemma 2. Let $X$ be an arbitrary Riemann surface. Suppose that $\varphi, \psi \in A(X)$, $\varphi \neq 0$, and $t$ is a real variable. Consider the two real-valued functions $f(t)=$ $\|\varphi+t \psi\|$ and $g(t)=\|\varphi+i t \psi\|$. Then the following two conditions are equivalent:

a. both $f(t)$ and $g(t)$ have second derivative at $t=0$,

b. $\frac{\psi^{2}}{\varphi} \in L^{1}(X)$.

Proof. Suppose first that $\frac{\psi^{2}}{\varphi} \in L^{1}(X)$. One verifies that $f^{\prime}(t)=\operatorname{Re} \iint \psi \frac{|\varphi+t \psi|}{\varphi+t \psi}$ provided that $\|\varphi+t \psi\| \neq 0$.

Therefore,

$$
\frac{f^{\prime}(t)-f^{\prime}(0)}{t}=\iint \operatorname{Re}\left(\frac{\psi}{t}\left(\frac{|\varphi+t \psi|}{\varphi+t \psi}-\frac{|\varphi|}{\varphi}\right)\right)=\iint \operatorname{Re}\left(\frac{\psi}{t} \frac{|\varphi|}{\varphi}\left(\frac{\left|1+t \frac{\psi}{\varphi}\right|}{1+t \frac{\psi}{\varphi}}-1\right)\right) .
$$

An easy calculation shows that, except at the zeros of $\varphi(z)$,

$$
\operatorname{Re}\left(\frac{\psi(z)}{t} \frac{|\varphi(z)|}{\varphi(z)}\left(\frac{\left|1+t \frac{\psi(z)}{\varphi(z)}\right|}{1+t \frac{\psi(z)}{\varphi(z)}}-1\right)\right)
$$

converges to

$$
\frac{\operatorname{Im}^{2}(\psi(z) \overline{\varphi(z)})}{|\varphi(z)|^{3}} \text { as } t \rightarrow 0
$$

Also

$$
\left|\operatorname{Re}\left(\frac{\psi}{t} \frac{|\varphi|}{\varphi}\left(\frac{\left|1+t \frac{\psi}{\varphi}\right|}{1+t \frac{\psi}{\varphi}}-1\right)\right)\right| \leq \frac{|\psi|}{|t|} 2|t| \frac{|\psi|}{|\varphi|} \in L^{1}(X)
$$

by Lemma 1.

Therefore, by Lebesque's Dominated Convergence Theorem, $\lim _{n \rightarrow \infty} \frac{f^{\prime}(t)-f^{\prime}(0)}{t}$ exists and is equal to $\iint_{X} \frac{(\operatorname{Im}(\psi \bar{\varphi}))^{2}}{|\varphi|^{3}}$. The same calculation shows that

$$
g^{\prime \prime}(0)=\iint_{X} \frac{(\operatorname{Im}(i \psi \bar{\varphi}))^{2}}{|\varphi|^{3}}=\iint_{X} \frac{(\operatorname{Re}(\psi \bar{\varphi}))^{2}}{|\varphi|^{3}} .
$$

Now suppose that both $f(t)$ and $g(t)$ have second derivative at $t=0$. For any subset $S$ of $X$, let $f_{S}(t)=\iint_{S}|\varphi+t \psi|$. Take any point $p$ in $X$. Let $m$ be the order of zero of $\varphi$ at $p$, and $k$ the order of zero of $\psi$ at $p$. Let $U$ be a small conformal disk with center at $p$ such that $\psi \varphi$ has no zeros in $\bar{U}-\{p\}$. Then, obviously, $f_{U^{c}}(t)=\iint_{U^{c}}|\varphi+t \psi|$ is a convex function, hence $f_{U^{c}}(t)-f_{U^{c}}(0)-t f_{U^{c}}^{\prime}(0) \geq 0$. If $m-k \geq 2+k$, then $f_{U}(t)-f_{U}(0)-t f_{U}^{\prime}(0)=C \epsilon(t)+o(\epsilon(t))$, where $C>0$, and 
$\epsilon(t)=|t|^{1+\frac{2+k}{m-k}}$ when $m-k>2+k$, while $\epsilon(t)=t^{2} \log \frac{1}{|t|}$ when $m-k=2+k$ (see [G2]).

Since $f=f_{U}+f_{U^{c}}$, the existence of $f^{\prime \prime}(0)$ yields $m-k<2+k$. Therefore $\frac{\psi^{2}}{\varphi} \in L_{l o c}^{1}(X)$.

Now take any compact set $K \subset X$. We have $\frac{\psi^{2}}{\varphi} \in L^{1}(K)$, and by the previous calculation

Since $f_{K^{c}}$ is convex,

$$
f_{K}^{\prime \prime}(0)=\iint_{K} \frac{(\operatorname{Im}(\psi \bar{\varphi}))^{2}}{|\varphi|^{3}}
$$

$$
\iint_{K} \frac{(\operatorname{Im}(\psi \bar{\varphi}))^{2}}{|\varphi|^{3}} \leq f^{\prime \prime}(0)
$$

Letting $K \rightarrow X$, we obtain

$$
\iint_{X} \frac{(\operatorname{Im}(\psi \bar{\varphi}))^{2}}{|\varphi|^{3}} \leq f^{\prime \prime}(0)<\infty
$$

Similarly we obtain

$$
\infty>g^{\prime \prime}(0) \geq \iint_{X} \frac{(\operatorname{Im}(i \psi \bar{\varphi}))^{2}}{|\varphi|^{3}}=\iint_{X} \frac{(\operatorname{Re}(\psi \bar{\varphi}))^{2}}{|\varphi|^{3}} .
$$

Adding (1) and (2), we see that

$$
\infty>\iint_{X} \frac{|\psi \bar{\varphi}|^{2}}{|\varphi|^{3}}=\iint_{X} \frac{|\psi|^{2}}{|\varphi|}
$$

Lemma 3. Let $X$ and $Y$ be arbitrary Riemann surfaces. Suppose that $L$ is a linear isometry from $A(X)$ onto $A(Y)$. Then, for every $\varphi$ and $\psi$ in $A(X)$,

$$
\frac{\psi^{2}}{\varphi} \in L^{1}(X) \quad \text { iff } \frac{L(\psi)^{2}}{L(\varphi)} \in L^{1}(Y) .
$$

Proof. Since $\|L(\varphi)+t L(\psi)\|=\|\varphi+t \psi\|$ and $\|L(\varphi)+i t L(\psi)\|=\|\varphi+i t \psi\|$, Lemma 3 is a trivial consequence of the equivalence of (a) and (b) in Lemma 2.

\section{Plane domain Case}

Definition 2. For any rational function

$$
R(z)=C \frac{\left(z-q_{1}\right)\left(z-q_{2}\right) \ldots\left(z-q_{m}\right)}{\left(z-p_{1}\right)\left(z-p_{2}\right) \ldots\left(z-p_{n}\right)}
$$

the order of $R$, denoted by $\operatorname{ord}(R)$, is equal to $m-n$. If $\operatorname{ord}(R)=-3$, then we say that $R$ has a pole at $\infty$.

Let $P$ be a set in the complex plane with parameter $z$. Let $R(P)$ be the set the of rational functions $r(z)$ which are holomorphic in $\mathbb{C}-P$, and for which $\|r\|=$ $\iint_{\mathbb{C}}|r(z)| d x d y<\infty$. The space $R(P)$ has several important properties:

1. The rational function $r(z)$ belongs to $L^{1}(\mathbb{C})$ if and only if all poles of $r(z)$ are simple and $\operatorname{ord}(r) \leq-3$. More generally, if $f(z)$ is holomorphic in a disc $D=\{z:|z-p|<\epsilon\}$ except for an isolated singularity at $p$ and $f$ belongs to $L^{1}(D)$, then the singularity of $f$ at $p$ is either removable or a simple pole. 
2. If the set $P$ is finite and consists of the $n \geq 3$ points $p_{1}, p_{2}, \ldots, p_{n-2}, a, b$, then the functions

$$
r_{j}(z)=\frac{1}{(z-a)(z-b)\left(z-p_{j}\right)}, \quad 1 \leq j \leq n-2,
$$

are a basis for $R(P)$.

3. Let $a, b$ be distinct points of $\mathbb{C}$ and let $\left\{p_{n}\right\}$ be a sequence in $\mathbb{C}-\{a, b\}$. Let $P(z)=(z-a)(z-b)$. If $p_{n} \rightarrow p \in \mathbb{C}-\{a, b\}$, then

$$
\frac{1}{P(z)\left(z-p_{n}\right)} \rightarrow \frac{1}{P(z)(z-p)}
$$

in $L^{1}(\mathbb{C})$.

We will frequently use an approximation theorem due to Bers ([B]) and Ahlfors $([\mathrm{A}])$. We refer to this theorem as the Bers' approximation theorem.

Theorem (Bers). If $X$ is a domain in the complex plane and $P$ is dense in $X^{c}$, the set complementary to $X$, then $R(P)$ is dense in $A(X)$.

Notice that if $\alpha$ is a conformal mapping from a plane domain $Y$ onto a plane domain $X$, then $\alpha^{*}: A(X) \rightarrow A(Y)$ defined by $\alpha^{*} \varphi(z)=\varphi(\alpha(z)) \alpha^{\prime}(z)^{2}$ is a complex linear isometry in the norms for $A(X)$ and $A(Y)$. Moreover if $\alpha$ is a Möbius transformation, then the set complementary to $X$ is transformed into the set complementary to $Y$.

In this section we prove:

Theorem 1. If $X$ and $Y$ are two plane domains such that $X^{c}$ is infinite and if $L: A(X) \rightarrow A(Y)$ is a linear invertible isometry, then there exist a constant $C$ of modulus one and a conformal mapping $\beta$ from $Y$ onto $X$ such that $L=C \beta^{*}$.

First we prove a lemma about rational functions which is used frequently in the proof of Theorem 1 .

Lemma 4. If $R_{1} \neq 0$ and $R_{2} \neq 0$ are two rational functions with no non-simple zeros in common, then there exist constants $C_{1}$ and $C_{2}$ such that

a. $C_{1} R_{1}+C_{2} R_{2}$ has no non-simple zeros,

b. if $R_{1}(p)=\infty$ or $R_{2}(p)=\infty$, then $C_{1} R_{1}(p)+C_{2} R_{2}(p)=\infty$, and

c. $\operatorname{ord}\left(C_{1} R_{1}+C_{2} R_{2}\right)=\max \left\{\operatorname{ord}\left(R_{1}\right), \operatorname{ord}\left(R_{2}\right)\right\}$.

Proof. Suppose $\left(C_{1}, C_{2}\right) \neq(0,0)$.

$C_{1} R_{1}(a)+C_{2} R_{2}(a)=0$ and $C_{1} R_{1}^{\prime}(a)+C_{2} R_{2}^{\prime}(a)=0$ imply that $R_{1}(a) R_{2}^{\prime}(a)-$ $R_{1}^{\prime}(a) R_{2}(a)=0$.

Consider $R(z)=R_{1}(z) R_{2}^{\prime}(z)-R_{1}^{\prime}(z) R_{2}(z)$. If $R=0$ then $R_{1}=C R_{2}$, and we can take $C_{1}=0, C_{2}=1$.

Suppose that $R \neq 0$. Then $A=\{a \mid R(a)=0\}$ is a finite set.

If $R_{1}(a), R_{2}(a), R_{1}^{\prime}(a)$ and $R_{2}^{\prime}(a)$ are all equal to zero, then $R_{1}$ and $R_{2}$ have a common non-simple zero at $a$.

If $a \in A$, and not both $R_{1}(a)$ and $R_{2}(a)$ are zero, then $C_{1} R_{1}(a)=-C_{2} R_{2}(a)$ is a linear condition for $C_{1}$ and $C_{2}$.

If $a \in A$, and not both $R_{1}^{\prime}(a)$ and $R_{2}^{\prime}(a)$ are zero, then $C_{1} R_{1}^{\prime}(a)=-C_{2} R_{2}^{\prime}(a)$ is a linear condition for $C_{1}$ and $C_{2}$.

Therefore, to satisfy condition (a), it is enough to take $\left(C_{1}, C_{2}\right)$ from the set $\left\{\left(C_{1}, C_{2}\right) \mid(\forall a \in A) \quad C_{1} R_{1}(a) \neq-C_{2} R_{2}(a)\right.$ or $\left.C_{1} R_{1}^{\prime}(a) \neq-C_{2} R_{2}^{\prime}(a)\right\}$. 
Since $R_{1}$ and $R_{2}$ have finitely many poles, to satisfy condition (b), we are only restricted by finitely many linear conditions for $C_{1}$ and $C_{2}$.

To satisfy (c), it is enough to take $C_{1} \neq 0$ and $C_{2} \neq 0$ such that $\alpha C_{1}+\beta C_{2} \neq 0$, where $\alpha$ and $\beta$ are such that

$$
R_{1}(z)=\alpha \frac{\left(z-a_{1}\right) \ldots\left(z-a_{n}\right)}{\left(z-b_{1}\right) \ldots\left(z-b_{m}\right)} \text { and } R_{2}(z)=\beta \frac{\left(z-c_{1}\right) \ldots\left(z-c_{k}\right)}{\left(z-d_{1}\right) \ldots\left(z-d_{j}\right)} .
$$

Therefore, to satisfy conditions (a), (b) and (c) we are only restricted by finitely many linear conditions for $C_{1}$ and $C_{2}$.

Proof of Theorem 1. Since the proof of Theorem 1 is rather long, it will be convenient to divide it into several steps.

Since $X^{c}$ and $Y^{c}$ are infinite, we may assume that $X$ and $Y$ are subsets of $\mathbb{C}-\{0,1\}$. Let $a$ belong to $X$. Our first goal is to find a point $b$ in $Y$ such that if $\varphi \in A(X)$ then $\varphi(a)=0$ if and only if $L \varphi(b)=0$. For that purpose we introduce two subsets of $A(X)$.

Definition 3. Let $F$ be the space of all differentials in $A(X)$ which have a nonsimple zero at $a$, and $V$ be the space of all differentials $\psi$ in $A(X)$ for which there exists a differential $\varphi$ in $F$ such that $\frac{\psi^{2}}{\varphi}$ is integrable in $X$;

$$
\begin{gathered}
F=\left\{\varphi \in A(X) \mid \varphi(a)=0 \text { and } \varphi^{\prime}(a)=0\right\}, \\
V=\bigcup_{\varphi \in F}\left\{\psi \in A(X) \mid \frac{\psi^{2}}{\varphi} \in L^{1}(X)\right\} .
\end{gathered}
$$

Step I. $\bar{V}$, the closure of $V$, is the set of all quadratic differentials in $A(X)$ that vanish at $a$. Therefore, $\operatorname{Codim}(\overline{L(V)})=1$.

Proof. If $\psi$ and $\varphi$ are in $A(X)$, and $\varphi$ belongs to $F$, and $\psi$ does not vanish at $a$, then $\frac{\psi^{2}}{\varphi}$ has a non-simple pole at $a$ and is not integrable. Therefore, $\bar{V}$ is contained in the set of quadratic differentials in $A(X)$ which vanish at $a$.

Conversely, assume that $\psi$ is a rational function in $A(X)$ and $\psi(a)=0$. Take $p \in X^{c}$ such that $p$ is not a pole of $\psi(z)$. Then $\varphi(z)=\frac{(z-a) \psi(z)}{z-p}$ is in $F$ and $\frac{\psi^{2}}{\varphi}=\frac{(z-p) \psi(z)}{z-a} \in L^{1}(X)$; thus, $\psi \in V$. By the Bers' approximation theorem, rational functions are dense in $A(X)$; thus, $\bar{V}=\{\psi \in A(X) \mid \psi(a)=0\}$.

If $\varphi_{0}$ is a quadratic differential in $A(X)$ such that $\varphi_{0}(a) \neq 0$, then $\varphi-\frac{\varphi(a)}{\varphi_{0}(a)} \varphi_{0}$ belongs to $\bar{V}$, for every $\varphi \in A(X)$. Therefore $\operatorname{Codim}(\overline{L(V)})=\operatorname{Codim}(L(\bar{V}))=$ $\operatorname{Codim}(\bar{V})=1$.

Step II. $L(V)=\bigcup_{\varphi \in L(F)}\left\{\psi \in A(Y) \mid \frac{\psi^{2}}{\varphi} \in L^{1}(Y)\right\}$.

Proof. Step II follows immediately from Lemma 3.

Let $p_{1}, p_{2}, \ldots, p_{5}$ be distinct points in $Y^{c}$. Since $\operatorname{Codim}(L(F))=2$,

$$
R_{0}=\frac{\alpha z^{2}+\beta z+\gamma}{\left(z-p_{1}\right) \ldots\left(z-p_{5}\right)} \in L(F) \text { for some }(\alpha, \beta, \gamma) \neq(0,0,0) .
$$

Hence, there is a rational function $R_{0}$ which is integrable over the complex plane and belongs to $L(F)$. Note that $R_{0}$ has at least three poles and at most two zeros. We may assume that $R_{0}$ has poles at 0,1 and $\infty$. To see that this can be done, let 
$p, q$, and $r$ be three distinct poles of $R_{0}$. If $\alpha$ is a Möbius transformation that maps 0 to $p, 1$ to $q$ and $\infty$ to $r$, then $\alpha^{*} \circ L$ is a linear isometry of $A(X)$ onto $A\left(\alpha^{-1}(Y)\right)$, and $\alpha^{*}\left(R_{0}\right)$ has poles at 0,1 and $\infty$.

In the next step we prove that $R_{0}$ has a double zero.

Step III. Every rational function $R$ in $L(F)$ with poles at 0,1 and $\infty$ has a nonsimple zero.

Proof. Suppose that some rational function $R$ in $L(F)$ has poles in 0,1 and $\infty$ and has only simple zeros.

If $\frac{1}{z(z-1)(z-p)} \in \overline{L(V)}$ for every $p \in Y^{c}-\{0,1\}$, then by property (2), every rational function in $A(Y)$ belongs to $\overline{L(V)}$. The Bers' approximation theorem implies $\overline{L(V)}=A(Y)$; a contradiction. Therefore, we may assume that $\frac{1}{z(z-1)(z-p)}$ is not in $\overline{L(V)}$ for some $p$ in $Y^{c}-\{0,1\}$.

If $p$ is a cluster point of $Y^{c}$, then by property (3) there exists a neighborhood $U$ of $p$ in $\mathbb{C}-\{0,1\}$ such that $\frac{1}{z(z-1)(z-q)}$ is not in $\overline{L(V)}$ for all $q$ in $U \cap Y^{c}$.

Take $a_{1}, a_{2}, a_{3} \in U \cap Y^{c}$. Since $\operatorname{Codim}(L(F))=2$, we can find three complex constants $\alpha, \beta$ and $\gamma$, not all equal to zero, such that

$$
M=\frac{\alpha z^{2}+\beta z+\gamma}{z(z-1)\left(z-a_{1}\right)\left(z-a_{2}\right)\left(z-a_{3}\right)} \in L(F) .
$$

Therefore, $M$ has a pole at $a_{1}, a_{2}$ or $a_{3}$. Assume that $M$ has a pole at $a_{1}$. Then, by Lemma 4, we can find constants $C_{1}$ and $C_{2}$ such that $T=C_{1} M+C_{2} R$ has poles at $0,1, \infty$ and $a_{1}$, and has no non-simple zeros. This implies $T \in L(F)$, and

$$
\frac{\left(\frac{1}{z(z-1)\left(z-a_{1}\right)}\right)^{2}}{T} \in L^{1}(Y)
$$

which contradicts the fact that $\frac{1}{z(z-1)\left(z-a_{1}\right)}$ is not in $\overline{L(V)}$.

Therefore, we may assume that $p$ is isolated in $Y^{c}$.

Now suppose that $\frac{1}{z(z-1)(z-q)} \in \overline{L(V)}$ for every $q$ in $Y^{c}-\{p, 0,1\}$. The reasoning in the second paragraph of this proof shows that $R\left(Y^{c}-\{p\}\right) \subset \overline{L(V)}$; therefore, by the Bers' approximation theorem and Step I, $\overline{L(V)}=\{\psi \in A(Y) \mid \psi$ is holomorphic at $p$.

Let $q_{1}, q_{2}, q_{3}$ and $q_{4}$ be four distinct points in the complement of $X$. Let $\varphi_{1}=$ $\frac{1}{\left(z-q_{1}\right)\left(z-q_{2}\right)\left(z-q_{3}\right)\left(z-q_{4}\right)}$ and $\varphi_{2}=(z-a) \varphi_{1}$. By Step I, $\varphi_{2}$ is in $\bar{V}$ and $\varphi_{1}$ is not in $\bar{V}$. That implies $L\left(\varphi_{2}\right)$ is in $\overline{L(V)}$, and $L\left(\varphi_{1}\right)$ is not in $\overline{L(V)}$; therefore, $L\left(\varphi_{2}\right)$ is holomorphic at $p$, and $L\left(\varphi_{1}\right)$ has a pole at $p$. This yields $\frac{\varphi_{1}^{2}}{\varphi_{2}} \in L^{1}(X)$, and $\frac{L\left(\varphi_{1}\right)^{2}}{L\left(\varphi_{2}\right)}$ has a non-simple pole at $p$, contradicting Lemma 3 .

Therefore, we may also assume that $\frac{1}{z(z-1)(z-q)}$ is not in $\overline{L(V)}$ with q isolated in $Y^{c}-\{0,1, p\}$. Since $F \subset V$, neither $\frac{1}{z(z-1)(z-p)}$ nor $\frac{1}{z(z-1)(z-q)}$ is in $\mathrm{L}(\mathrm{F})$.

Take $r \in Y^{c}-\{0,1, p, q\}$. Since $\operatorname{Codim}(L(F))=2$, there exist three constants $\alpha, \beta$ and $\gamma$, not all equal to zero, such that

$$
M=\frac{\alpha z^{2}+\beta z+\gamma}{z(z-1)(z-p)(z-q)(z-r)} \in L(F) .
$$

If $\frac{1}{z(z-1)(z-r)}$ is not in $L(F)$, then $M$ has a pole at $p$ or at $q$. Assume that $M$ has a pole at $p$. By Lemma 4 , we can find constants $C_{1}$ and $C_{2}$ such that $C_{1} M+C_{2} R$ 
has poles at $p, 0,1, \infty$, and has no non-simple zeros. But then

$$
\frac{\left(\frac{1}{z(z-1)(z-p)}\right)^{2}}{C_{1} M+C_{2} R} \in L^{1}(Y)
$$

thus, $\frac{1}{z(z-1)(z-p)}$ is in $L(V)$, a contradiction.

Therefore $\frac{1}{z(z-1)(z-r)} \in L(F)$ for all $\mathrm{r}$ in $Y^{c}-\{\infty, 0,1, p, q\}$, and by property (2) and the Bers' approximation theorem, $L(F)=\{\psi \in A(Y) \mid \psi$ is holomorphic at $p$ and $q\}$. If $\psi \in L(F)$ and $\varphi \in A(Y)-L(F)$, then $\frac{\varphi^{2}}{\psi}$ has a non-simple pole at $p$ or $q$. Therefore $L(F)=L(V)$, a contradiction.

Step IV. $L(F)$ is the set of all differentials in $A(Y)$ with a non-simple zero at some point $b$ in $Y$.

Proof. The rational function $R_{0}$ introduced right after Step II belongs to $L(F)$ and has poles at 0,1 and $\infty$; thus by Step III it has a double zero at some point $b$. Note that $b$ is the only zero of $R_{0}$. Suppose that $R$ is a rational function in $A(Y)$ with a non-simple zero at $b$. Let $R(z)=(z-b)^{2+k} R_{1}(z)$ with $k \geq 0$, and $R_{1}(b)$ is neither 0 nor $\infty$. Then, since $\operatorname{Codim}(L(F))=2$,

$$
P(z)=R_{1}(z)\left[\alpha(z-b)^{2+k}+\beta(z-b)+\gamma\right] \in L(F)
$$

for some $(\alpha, \beta, \gamma) \neq 0$. If $P(b) \neq 0$ or $P^{\prime}(b) \neq 0$, then, by Lemma 4 , we can find constants $C_{1}$ and $C_{2}$ such that $C_{1} P+C_{2} R_{0}$ has poles at $0,1, \infty$, and has no nonsimple zeros. This contradicts Step III. Therefore $P(b)=0$ and $P^{\prime}(b)=0$. This yields $\gamma=0$ and $\beta=0$, which means $R \in L(F)$. Therefore, every rational function in $A(Y)$ with a non-simple zero at $b$ is in $L(F)$. Suppose that $b \in Y^{C}$. Choose four distinct points $p_{1}, p_{2}, p_{3}$ and $p_{4}$ in $Y^{c}-\{0,1, b\}$. Then, since $\operatorname{Codim}(F)=2$,

$$
M=\frac{\alpha+\beta z+\gamma z^{2}}{(z-b)\left(z-p_{1}\right) \ldots\left(z-p_{4}\right)} \in L(F) \text { for some }(\alpha, \beta, \gamma) \neq 0 .
$$

Since $b$ is the only zero of $R_{0}$, by Lemma 4 we can find constants $C_{1}$ and $C_{2}$ such that $C_{1} M+C_{2} R_{0}$ has poles at $0,1, \infty$ and has no non-simple zeros, again contradicting Step III.

By the Bers' approximation theorem and the fact that $\operatorname{Codim}(L(F))=2$, we have $L(F)=\{\psi \in A(Y) \mid \psi$ has a non-simple zero at $b\}$.

Step $\mathrm{V}$. There exists a bijection $\beta$ from $Y$ onto $X$ such that

$$
\psi(\beta p)=0 \text { iff } L \psi(p)=0 \text {, for every } p \text { in } Y \text {. }
$$

Proof. Steps II and IV imply that every $\psi$ in $L(V)$ has a zero at $b$. Therefore, by Step I, $\overline{L(V)}=\{\psi \in A(Y) \mid \psi(b)=0\}$.

Therefore, for every $\psi$ in $A(X)$,

$$
\psi(a)=0 \Leftrightarrow L(\psi)(b)=0 .
$$

Step V follows by applying previous steps to $L^{-1}$.

Remark. In Step I we proved that $\operatorname{Codim}(\overline{L(V)})=1$; in Step III we constructed functions $L\left(\varphi_{1}\right)$ and $L\left(\varphi_{2}\right)$ such that $L\left(\varphi_{2}\right)$ is in $\overline{L(V)}, L\left(\varphi_{1}\right)$ is not in $\overline{L(V)}$, and $\frac{L\left(\varphi_{1}\right)^{2}}{L\left(\varphi_{2}\right)} \in L^{1}(Y)$. In Step II we proved that

$$
\overline{L(V)}=\overline{\bigcup_{\varphi \in S}\left\{\psi \in A(Y) \mid \frac{\psi^{2}}{\varphi} \in L^{1}(Y)\right\}},
$$


where $S=L(F)$ is a closed subspace of codimension 2 in $A(Y)$. A close look in the proofs of Steps I, III and IV shows that these three properties of a closed subspace $\overline{L(V)}$ are sufficient to establish that $\overline{L(V)}=\{\psi \in A(Y) \mid \psi(b)=0\}$, for some $b \in Y$. Therefore we have proved the following theorem.

Theorem. Let $X$ be a plane domain such that $X^{c}$ is infinite, and let $E$ be a closed subspace of $A(X)$. Then there exists a point a in $X$ such that $E=\{\psi \in$ $A(X) \mid \psi(a)=0\}$ if and only if the following three conditions hold:

1. E has codimension 1,

2. there exist a differential $\varphi$ in $E$ and a differential $\psi$ in $A(X)-E$ such that $\frac{\psi^{2}}{\varphi} \in L^{1}(X)$, and

3. there is a closed subspace $F$ of $A(X)$ such that $F$ has codimension 2 and $E=\overline{\bigcup_{\varphi \in F}\left\{\psi \in A(X) \mid \frac{\psi^{2}}{\varphi} \in L^{1}(X)\right\}}$.

The author is thankful to the referee for pointing out this remark.

Step VI. $\beta$ is a conformal homeomorphism.

Proof. Let $\varphi(z)=\frac{1}{\left(z-q_{1}\right) \ldots\left(z-q_{4}\right)}, \quad p \in Y$, and $\beta(p)=q$. The function

$$
z \rightarrow \frac{z-q}{\left(z-q_{1}\right) \ldots\left(z-q_{4}\right)}
$$

is in $A(X)$ and has a zero at $q$. Hence,

$$
L(z \varphi)(p)-q L(\varphi)(p)=0 .
$$

Thus, $\beta(p)=\frac{L(z \varphi)(p)}{L(\varphi)(p)}$. Therefore, $\beta$ is holomorphic.

Step VII. $L=C \beta^{*}$ for some complex constant $C$ of modulus one.

Proof. Without loss of generality we can assume that $X=Y$ and $\beta \equiv i d$. If we let $\varphi(z)=\frac{1}{\left(z-q_{1}\right) \ldots\left(z-q_{4}\right)}$, then for every $\psi \in A(X)$ and every $p \in X$, the quadratic differential $\psi-\frac{\psi(p)}{\varphi(p)} \varphi$ is in $A(X)$ and has a zero at $p$. Therefore, by Step $\mathrm{V}, L \psi(p)-\frac{\psi(p)}{\varphi(p)} L \varphi(p)=0$. This yields $L \psi(p)=\psi(p) \frac{L \varphi(p)}{\varphi(p)}$. Let $f=\frac{L \varphi}{\varphi}$. Then $L \psi=f \psi \quad$ for all $\psi \in A(X)$.

The family $\left\{\psi, L \psi, L^{2} \psi, \ldots\right\}$ is normal. Therefore, $|f| \leq 1$. Since $\|\psi\|=\|L \psi\|$, $|f(z)|=1$ for every $z$; thus $f$ is a constant function.

\section{Riemann SURFaces of finite Genus}

For any subset $\Lambda$ of any Riemann surface $W$ define $R(W, \Lambda)$ to be the set of meromorphic quadratic differentials $\varphi$ on $W$ such that $\int_{W}|\varphi|<\infty$ and $\varphi$ has only finitely many poles, all of them in $\Lambda$. We now prove a version of Bers' approximation theorem that holds for general Riemann surfaces. We refer to this theorem as the generalized Bers' approximation theorem.

Theorem. Let $V$ be any open subset of $W$. If $\Lambda$ is dense in $W-V$, then $R(W, \Lambda)$ is dense in $A(V)$.

Proof. If $W-V$ is finite, then $\Lambda=W-V$ and the theorem follows from property (1) in Section 2. If $W-V$ is infinite, fix three points $p_{1}, p_{2}$ and $p_{3}$ in $W-V$ and let $W_{1}=W-\left\{p_{1}, p_{2}, p_{3}\right\}$. Let $\pi: \Delta \rightarrow W_{1}$ be the universal covering map with a covering group $\Gamma$. Let $U=\pi^{-1}(V)$, and let $\Theta: A(U) \rightarrow A(U, \Gamma)=A(V)$ be the 
Poincaré theta series. Let $B=\pi^{-1}(\Lambda)$. Since $\Lambda$ is dense in $W-V, B$ is dense in $\Delta-U$. By the Bers' approximation theorem, $S(B)$, the space of rational functions which are integrable over the complex plane and holomorphic in $\Delta-B$, is dense in $A(U)$. Since $\Theta$ is surjective (see $[\mathrm{K}]), \Theta(S(B))$ is dense in $\Theta(A(U))=A(U, \Gamma)=$ $A(V)$. Every quadratic differential in $\Theta(S(B))$ is integrable in $W$, and holomorphic in $W$ except for possibly finitely many poles in $\Lambda$; thus it belongs to $R(W, \Lambda)$.

Assume both $X$ and $Y$ are Riemann surfaces which are subsets of compact Riemann surfaces $X_{1}$ and $Y_{1}$, respectively. Let $L$ be a linear isometry from $A(X)$ onto $A(Y)$. If $X_{1}-X$ is a finite set, then $A(X)$ is finite dimensional; thus, $A(Y)$ is also finite dimensional; so $Y_{1}-Y$ contains only finitely many points. In that case, all holomorphic automorphisms between Teichmüller spaces of $X$ and $Y$ are determined in [EK]. Therefore, we assume that $X_{1}-X$ and $Y_{1}-Y$ are infinite sets.

An example for $X$ is a Riemann surface of finite topological type with non-empty border.

Denote $R\left(X_{1}, X_{1}-X\right)$ by $R(X)$, and $R\left(Y_{1}, Y_{1}-Y\right)$ by $R(Y)$. Hence, for every $\varphi$ in $R(X)$ we can find a neighborhood $U$ of $X$ so that all poles of $\varphi$ in $U$ belong to $\bar{X}-X$.

In this section we prove our main result:

Theorem 2. If both $X$ and $Y$ are Riemann surfaces which are subsets of compact Riemann surfaces $X_{1}$ and $Y_{1}$, respectively, and the set $X_{1}-X$ has infinitely many points, then every linear isometry from $A(X)$ onto $A(Y)$ comes from a pull-back by conformal mapping from $Y$ onto $X$ and multiplication by a complex constant of modulus one.

In the proof of Theorem 2 we use a theorem due to Noether about the existence of meromorphic functions with certain properties on a compact Riemann surface. We refer to this theorem as the "gap" theorem.

The "Gap" Theorem (Noether). Let $M$ be a compact Riemann surface of genus $g$. For any points $b_{1}, b_{2}, b_{3}, \ldots, b_{n}$ (not necessarily distinct) in $M$ with $n \geq 2 g$, there exists a meromorphic function $f$ on $M$ such that Divisor $(f)$ is a multiple of the divisor $-\chi_{b_{1}}-\chi_{b_{2}}-\ldots-\chi_{b_{n-1}}-\chi_{b_{n}}$ and Divisor $(f)$ is not a multiple of the divisor $-\chi_{b_{1}}-\chi_{b_{2}}-\ldots-\chi_{b_{n-1}}$.

The proof of the "gap" theorem can be found in [FK].

The proof of Theorem 2 will follow the methods developed in Section 2. Before we begin to prove Theorem 2, we prove two preliminary lemmas. First we adapt Lemma 4 to a new situation.

Lemma 5. If $R_{1}, R_{2} \in R(X)$ are non-zero meromorphic quadratic differentials with no non-simple zeros in common on $\bar{X}$, then there exist constants $C_{1}$ and $C_{2}$ such that

a. $C_{1} R_{1}+C_{2} R_{2}$ has no non-simple zeros in $\bar{X}$, and

b. if $p \in \bar{X}$ is a pole of $R_{1}$ or $R_{2}$, then $p$ is a pole of $C_{1} R_{1}+C_{2} R_{2}$.

Proof. Take a finite open cover $\left\{V_{i}\right\}$ of $\bar{X}$ such that $\overline{V_{i}} \subset U_{i}$ and $z_{i}: U_{i} \rightarrow z_{i}\left(U_{i}\right)$ are charts in $X_{1}$. Define $P_{i}: z_{i}\left(U_{i}\right) \rightarrow \overline{\mathbb{C}}$ as $P_{i}=R_{1} R_{2}^{\prime}-R_{1}^{\prime} R_{2}$ in terms of the local parameter $z_{i}$. Then $A=\bigcup_{i}\left\{a \in V_{i} \mid P_{i}\left(z_{i}(a)\right)=0\right\}$ is a finite set provided that $R_{1}$ and $R_{2}$ are linearly independent, and the rest of the proof is the same as in Lemma 4. 
The second preliminary lemma is a consequence of the "gap" theorem and Lemma 5 .

Lemma 6. Let $S$ be an infinite subset of the compact Riemann surface $N$. Let $s_{1}, s_{2}, \ldots, s_{n} \in S$ and $m \in N-S$. Then there exists a meromorphic function $g$ on $N$, such that

a. $g(m)=0$,

b. all poles of $g$ are simple and belong to $S-\left\{s_{1}, s_{2}, \ldots, s_{n}\right\}$,

c. $g$ has no zeros in $\left\{s_{1}, s_{2}, \ldots, s_{n}\right\}$, and

d. all zeros of $g$ in $N$ are simple.

Proof. Since $S$ is an infinite set, by the "gap" theorem, there exist two linearly independent meromorphic functions $h_{1}$ and $h_{2}$ on $N$ which both satisfy (b). Let $f$ be a non-trivial linear combination of $h_{1}$ and $h_{2}$ such that $f(m)=0$. Let $\left\{a_{1}=\right.$ $\left.m, a_{2}, a_{3}, \ldots, a_{k}\right\}$ be the set of all zeros of $f$ in $N$. Let $m_{i}$ be the order of zero of $f$ at $a_{i}$ for $i>1$, and let $m_{1}+1$ be the order of zero of $f$ at $a_{1}$. By the "gap" theorem, there exist meromorphic functions $f_{i}$ on $N$ such that $f_{i}$ has a pole of order $m_{i}$ at $a_{i}$, all poles of $f_{i}$ in $N-\left\{a_{i}\right\}$ are simple and belong to $S-\left\{s_{1}, \ldots, s_{n}\right\}$, and such that $f$ and $f_{i}$ have no poles in common. The function $f f_{i}$ satisfies (a) and (b), and $f f_{i}\left(a_{i}\right) \neq 0$, for $i>1$, and $f f_{1}$ has a simple zero at $m$. By induction, we can find constants $\alpha_{1}, \ldots, \alpha_{k}$ such that the meromorphic function $h=\alpha_{1} f f_{1}+\alpha_{2} f f_{2}+\ldots+\alpha_{k} f f_{k}$ has a simple zero at $m$ and has no zeros in $\left\{a_{2}, a_{3}, \ldots, a_{k}\right\}$. Now $f$ and $h$ have no non-simple zeros in common, and by the proof of Lemma 5, some linear combination $g=\alpha f+\beta h$ satisfies (d) and (c). (Note that $g\left(s_{i}\right)=0$ is equivalent to $\alpha f\left(s_{i}\right)+\beta h\left(s_{i}\right)=0$, a linear condition for $\alpha$ and $\beta$ ). Since both $f$ and $h$ satisfy (a) and (b), $g$ satisfies (a),(b),(c) and (d).

Now we begin to prove Theorem 2 .

First we define spaces $F$ and $V$ in the same way as in Section 2 .

Definition 4. Choose a point $b \in X$. Let

$$
F=\left\{\varphi \in A(X) \mid \varphi(b)=0 \text { and } \varphi^{\prime}(b)=0\right\}
$$

and

$$
V=\bigcup_{\varphi \in F}\left\{\psi \in A(X) \mid \frac{\psi^{2}}{\varphi} \in L^{1}(X)\right\} .
$$

Step I. $\bar{V}$ is the space of all differentials in $A(X)$ that vanish at $b$.

Proof. If $\psi$ does not vanish at $b$ and $\varphi$ is in $F$, then $\frac{\psi^{2}}{\varphi}$ has a non-simple pole at $b$. That proves that $\bar{V}$ is a subset of the space of all differentials in $A(X)$ that vanish at $b$.

To prove the converse, suppose that $R \in R(X)$ is such that $R(b)=0$.

The set $X_{1}-X$ is infinite; thus, by Lemma 6 , we can choose a meromorphic function $g$ on $X_{1}$ such that

a. $g(b)=0$,

b. all poles of $g$ are simple and belong to $X_{1}-X$,

c. $g$ and $R$ have no poles in common,

d. All zeros of $g$ are simple, and

e. $g$ has no zeros at the poles of $R$. 
The quadratic differential $\varphi=g R$ has a non-simple zero at $b$, and all poles of $g$ and $R$ are simple, distinct, and outside $X$. Hence $\varphi$ is in F. Futhermore, $\frac{R^{2}}{\varphi}=\frac{R}{g} \in L^{1}(X)$; thus, $R \in V$. Since $R(X)$ is dense in $A(X)$, we have $\bar{V}=\{\psi \in$ $A(X) \mid \psi(b)=0\}$.

Step II. $L(V)=\bigcup_{\varphi \in L(F)}\left\{\psi \in A(Y) \mid \frac{\psi^{2}}{\varphi} \in L^{1}(Y)\right\}$.

Proof. Step II follows immediately from Lemma 3.

Step III. Let $p$ be an isolated point in $Y_{1}-Y$. Then $\overline{L(V)} \neq\{\psi \in A(Y) \mid \psi$ is holomorphic at $p$.

Proof. Suppose that $\overline{L(V)}=\{\psi \in A(Y) \mid \psi$ is holomorphic at $p\}$.

Let $\varphi \in R(X)$ be such that $\varphi(b) \neq 0$. Such a differential exists by an easy consequence of the "gap" theorem.

By Lemma 6, we can choose a meromorphic function $g$ on $X_{1}$ such that

a. $g(b)=0$,

b. all poles of $g$ are simple and belong to $X_{1}-X$,

c. $g$ and $\varphi$ have no poles in common,

d. all zeros of $g$ are simple, and

e. $g$ has no zeros at the poles of $\varphi$.

Therefore $\varphi$ is not in $\bar{V}$, and $g \varphi$ is in $\bar{V}$; thus $L(\varphi)$ has a pole at $p$ and $L(g \varphi)$ is holomorphic at $p$. Hence $\frac{\varphi^{2}}{g \varphi}=\frac{\varphi}{g} \in L^{1}(X)$, and $\frac{L(\varphi)^{2}}{L(g \varphi)}$ has a non-simple pole at $p$, contradicting Lemma 3.

As another easy consequence of the "gap" theorem, for any $c$ in $Y_{1}-Y$ there is a $\varphi$ in $R(Y)$ with a simple pole at $c$. Let $c_{1}, c_{2}$, and $c_{3}$ be distinct points of $Y_{1}-Y$, and let $Q(Y)$ be the set of $\varphi$ in $R(Y)$ that have simple poles at $c_{1}, c_{2}$, and $c_{3}$. Since $R(Y)$ is dense in $A(Y)$, so is $Q(Y)$.

Step IV. Every $R$ in $Q(Y) \cap L(F)$ has a non-simple zero in $\bar{Y}$.

Proof. Suppose that some $R \in Q(Y) \cap L(F)$ has only simple zeros in $\bar{Y}$. If all poles (in $Y_{1}$ ) of some $\psi \in R(Y)$ are in $\left\{c_{1}, c_{2}, c_{3}\right\}$, then $\frac{\psi^{2}}{R} \in L^{1}(Y)$; thus $\psi$ belongs to $L(V)$. Let $Y_{0}=Y_{1}-\left\{c_{1}, c_{2}, c_{3}\right\}$. For every $p \in Y_{0}-Y$, take $\psi_{p} \in A\left(Y_{0}-\{p\}\right)-A\left(Y_{0}\right)$. The existence of such a differential is guaranteed by the fact that the dimension of the space of integrable holomorphic quadratic differentials on a Riemann surface of finite analytic type $(g, n)$ is $3 g-3+n$ whenever $n>2$; and it can be easily constructed using the Poincaré theta series. If $\psi_{p}$ is in $\overline{L(V)}$ for every $p \in Y_{0}-Y$, then $R(Y) \subset \overline{L(V)}$, a contradiction. Therefore $\psi_{p}$ is not in $\overline{L(V)}$ for some $p \in$ $Y_{0}-Y$.

If $p$ is a cluster point of $Y_{1}-Y$, then take a sequence $p_{n} \in Y_{0}-Y-\{p\}$ such that $p_{n} \rightarrow p$. By the generalized Bers' approximation theorem, $R\left(Y_{0},\left\{p_{1}, p_{2}, \ldots\right\}\right)$ is dense in $A\left(Y_{0}-\left\{p, p_{1}, p_{2}, \ldots\right\}\right)$. Therefore, there exists a sequence $\left(\psi_{n}\right)$ in $A(Y)$ such that $\psi_{n} \rightarrow \psi_{p}$, and $\psi_{n}$ is holomorphic in $Y_{0}$ except for finitely many poles in $\left\{p_{1}, p_{2}, p_{3}, \ldots\right\}$. Without loss of generality, we can assume that $\psi_{n}$ and $\psi_{m}$ have no poles in common on $Y_{0}$ for $m \neq n$, and that $\psi_{n}$ is not in $\overline{L(V)}$ for every n. Since $\operatorname{Codim}(F)=2$, there exist constants $\alpha, \beta$ and $\gamma$, not all equal to 0 , such that $M=\alpha \psi_{1}+\beta \psi_{2}+\gamma \psi_{3} \in L(F)$. Assume $\alpha \neq 0$. Then by Lemma 5 , we can find constants $C_{1}$ and $C_{2}$ such that $C_{1} R+C_{2} M$ belongs to $Q(Y)$, has poles wherever 
$\psi_{1}$ has poles, and has no non-simple zeros in $\bar{Y}$. That yields $\frac{\psi_{1}^{2}}{C_{1} R+C_{2} M} \in L^{1}(Y)$, a contradiction that proves that $p$ must be isolated in $Y_{1}-Y$.

Suppose that $\psi_{q}$ is in $\overline{L(V)}$ for every $q \in Y_{0}-Y-\{p\}$. Then, since $\operatorname{Codim}(\bar{V})=1$, we have $\overline{L(V)}=\{\psi \in A(Y) \mid \psi$ is holomorphic at $p\}$, which contradicts Step III.

Therefore, $\psi_{q}$ is not in $\overline{L(V)}$ for some $q$ isolated in $Y_{0}-Y-\{p\}$. This implies that neither $\psi_{q}$ nor $\psi_{p}$ is in $L(F)$. Let $\psi \in R(Y)$ be holomorphic at $p$ and $q$. Suppose that $\psi$ does not belong to $L(F)$. Since $\operatorname{Codim}(F)=2$, there exist constants $\alpha, \beta$ and $\gamma$, not both $\alpha$ and $\beta$ equal to zero, such that $M=\alpha \psi_{p}+\beta \psi_{q}+\gamma \psi \in L(F)$. Assume that $\alpha \neq 0$. Then $M$ has a pole at $p$, and, by Lemma 5 , there exist constants $C_{1}$ and $C_{2}$ such that $T=C_{1} M+C_{2} R$ has poles at $p, c_{1}, c_{2}, c_{3}$ and has no non-simple zeros. But then $\frac{\psi_{p}^{2}}{T} \in L^{1}(Y)$. This contradiction proves that every $\psi \in R(Y)$ which is holomorphic at $p$ and $q$ belongs to $L(F)$. Since $R(Y)$ is dense in $A(Y)$ and $\operatorname{Codim}(F)=2$, we have

$$
L(F)=\{\psi \in A(Y) \mid \psi \text { is holomorphic at } p \text { and } q\} .
$$

If $\psi \in L(F)$, and $\varphi$ is not in $L(F)$, then $\frac{\varphi^{2}}{\psi}$ has a non-simple pole at $p$ or $q$. Thus $L(F)=L(V)$; a contradiction.

Step V. There exists a point $a$ in $Y$ such that every $R$ in $L(F) \cap R(Y)$ has a non-simple zero at $a$.

Proof. Take a differential $R$ in $L(F) \cap Q(Y)$. This is possible by changing $c_{1}, c_{2}$ and $c_{3}$ in the definition of $Q(Y)$ if necessary. Let $\left\{a_{1}, a_{2}, \ldots, a_{n}\right\}$ be the set of all non-simple zeros of $R$ in $\bar{Y}$. If there exists a differential $R_{1}$ in $L(F) \cap R(Y)$ such that $a_{1}$ is not a non-simple zero of $R_{1}$, then by the proof of Lemma 5 , there exist constants $C_{1}$ and $C_{2}$ such that the set of non-simple zeros of $C_{1} R_{1}+C_{2} R$ in $\bar{Y}$ is a subset of $\left\{a_{2}, a_{3}, \ldots, a_{n}\right\}$, and $C_{1} R_{1}+C_{2} R$ belongs to $Q(Y)$. By induction, there exists $a \in \bar{Y}$ such that every quadratic differential in $L(F) \cap R(Y)$ has a non-simple zero at $a$.

We now show that $a$ must be in $Y$. Suppose not. Then $a \in \bar{Y}-Y$; thus there exists a quadratic differential $\psi_{a}$ in $R(Y)$ which has a pole at $a$. By an easy consequence of the "gap" theorem, there exist differentials $\psi$ and $\varphi$ in $R(Y)$ such that $\psi(a) \neq 0, \varphi(a)=0$, and $\varphi^{\prime}(a) \neq 0$, Therefore, $\operatorname{Codim}(L(F))=2$ implies that some non-trivial linear combination of $\varphi, \psi$ and $\psi_{a}$ belongs to $L(F)$, thus has a non-simple zero at $a$; a contradiction.

Step VI. $L(F)$ is the space of all quadratic differentials in $A(Y)$ with a non-simple zero at $a$.

Proof. Let $\varphi$ and $\psi$ be the quadratic differentials introduced toward the end of the proof of Step V. Take $R$ in $R(Y)$ such that $R$ has a non-simple zero at $a$. Since $\operatorname{Codim}(F)=2$, some linear combination of $\psi, \varphi$ and $R$ is in $L(F)$, thus has a nonsimple zero at $a$. That implies that $R$ is in $L(F)$. Since $R(Y)$ is dense in $A(Y)$, the set of all differentials in $A(Y)$ with a non-simple zero at $a$ is a subset of $L(F)$. Since $\operatorname{Codim}(F)=2$, we have $L(F)=\left\{\psi \in A(Y) \mid \psi(a)=0\right.$ and $\left.\psi^{\prime}(a)=0\right\}$.

Step VII. $\overline{L(V)}$ is the set of all differentials in $A(Y)$ that vanish at $a$.

Proof. As in Section 2, Step VII follows immediately from Steps I, II and VI.

Remark. The proofs of Steps I-VII generalize the theorem from the remark in Section 2 . 
Theorem. Let $X$ be a Riemann surface of finite genus and infinite analytic type and let $E$ be a closed subspace of $A(X)$. Then there exists a point a in $X$ such that $E=\{\psi \in A(X) \mid \psi(a)=0\}$ if and only if the following three conditions hold:

1. E has codimension 1,

2. there exist a differential $\varphi$ in $E$ and a differential $\psi$ in $A(X)-E$ such that $\frac{\psi^{2}}{\varphi} \in L^{1}(X)$, and

3. there is a closed subspace $F$ of $A(X)$ such that $F$ has codimension 2 and $E=\overline{\bigcup_{\varphi \in F}\left\{\psi \in A(X) \mid \frac{\psi^{2}}{\varphi} \in L^{1}(X)\right\}}$.

Notice that by Lemma 3 , the properties 1, 2, and 3 of a closed set $E$ are equivalent under any linear isometry of $A(X)$. The next step is a consequence of this invariance.

Step VIII. There exists a bijection $\beta$ from $X$ onto $Y$ such that $\psi(p)=0$ iff $L \psi(\beta(p))=0$ for every $p$ in $X$ and every $\psi$ in $A(X)$.

Proof. To prove Step VIII, consider $L^{-1}$ and observe that by an easy consequence of the "gap" theorem, for any two distinct points $p$ and $q$ in $X$, there exists a differential $\varphi$ in $A(X)$ such that $\varphi(p)=0$ and $\varphi(q) \neq 0$.

Step IX. $\beta$ is continuous.

Proof. Suppose that $\beta$ is not continuous. Then there exist a point $p$ and a sequence $\left(p_{n}\right)$ in $X$ that converges to $p$ such that $\beta\left(p_{n}\right)$ does not converge to $\beta(p)$. Since $\bar{Y}$ is compact, we can assume that $\beta\left(p_{n}\right)$ converges to a point $q$ in $\bar{Y}-\{\beta(p)\}$.

By Lemma 6, there exist a quadratic differential $\psi \in A(Y)$ and a function $g$, meromorphic in $Y_{1}$ and holomorphic in $Y$, such that $g \psi \in A(Y), g(\beta(p)) \neq$ $0, \psi(\beta(p)) \neq 0$, and $g(q)=0$. Then $\varphi_{n}=\left(g-g\left(\beta\left(p_{n}\right)\right)\right) \psi$ belongs to $A(Y)$ and vanishes at $\beta\left(p_{n}\right)$. Furthermore, $\varphi_{n}$ converges to $g \psi$ in the $L^{1}-$ norm. Therefore $\left\|L^{-1}\left(\varphi_{n}\right)-L^{-1}(g \psi)\right\| \rightarrow 0, L^{-1}\left(\varphi_{n}\right)\left(p_{n}\right)=0$, and $L^{-1}(g \psi)(p) \neq 0$. This contradicts the principle of argument.

Step X. $\beta$ is holomorphic.

Proof. Take any two linearly independent quadratic differentials $\varphi$ and $\psi$ in $R(X)$. Fix $p \in X$. The quadratic differential $\varphi(p) \psi-\psi(p) \varphi$ has a zero at $p$. Step VIII implies that $\varphi(p) L(\psi)-\psi(p) L(\varphi)$ has a zero at $\beta(p)$. Therefore $\frac{L \psi}{L \varphi}(\beta(p))=\frac{\psi}{\varphi}(p)$ for almost every $p$. Since $\beta$ is continuous and $\frac{L \psi}{L \varphi}$ is meromorphic,

$$
\beta(p)=\left(\frac{L \psi}{L \varphi}\right)^{-1} \circ\left(\frac{\psi}{\varphi}\right)(p),
$$

locally, for almost every $p$; thus $\beta$ is holomorphic.

Step XI. There exists a constant $C$ of modulus one such that $L=C\left(\beta^{-1}\right)^{*}$.

Proof. By taking a geometric postcomposition, we can assume that $\beta=i d$.

The proof of Step X implies that $L(\psi)=\psi \frac{L(\varphi)}{\varphi}$. Since $\{\psi, L(\psi), L(L(\psi)), \ldots\}$ is a normal family, $\left|\frac{L \varphi}{\varphi}\right| \leq 1$. Also ||$L(\psi)\|=\| \psi \|$, and thus $\left|\frac{L \varphi}{\varphi}\right|=1$. 


\section{Some APplichtions to Teichmüller SPACES}

In [EG], Earle and Gardiner say that a Riemann surface $X$ has the isometry property if for all Riemann surfaces $Y$ and $W$ that are quasiconformally equivalent to $X$ every linear isometry $L$ of $A(Y)$ onto $A(W)$ equals $C \alpha^{*}$ for some conformal mapping $\alpha$ of $W$ onto $Y$ and some complex constant $C$ of modulus 1 .

Theorem. If the Riemann surface $X$ is a subset of a compact Riemann surface $X_{1}$ and the set $X_{1}-X$ is infinite, then $X$ has the isometry property.

Proof. This follows trivially from Theorem 2 because if $Y$ is quasiconformally equivalent to $X$ there is a compact Riemann surface $Y_{1}$ (quasiconformally equivalent to $\left.X_{1}\right)$ such that $Y \subset Y_{1}$ and $Y_{1}-Y$ is infinite. $X$.

This result allows us to apply the theorems in $[\mathrm{EG}]$ to the Teichmüller space of

The points in the Teichmüller space Teich $(X)$ of a Riemann surface $X$ are equivalence classes $[f: X \rightarrow Y]$ of quasiconformal mappings with domain $X$, and the basepoint of $T$ eich $(X)$ is the equivalence class $[i d]$ of the identity map. The tangent space to $T e i c h(X)$ at its basepoint is $A(X)^{*}$ and the infinitesimal Kobayashi metric on $A(X)^{*}$ is the standard dual norm. For any quasiconformal homeomorphism $g$ of $X$ onto $Y$, right translation by $g^{-1}$ induces a biholomorphic map $\rho_{g}$ from Teich $(X)$ onto Teich $(Y): \rho_{g}([f])=\left[f \circ g^{-1}\right]$. We call the bijection $\rho_{g}$ a geometric isomorphism.

Theorem 3. Let the Riemann surfaces $X$ and $Y$ be the subsets of compact Riemann surfaces $X_{1}$ and $Y_{1}$, respectively, such that the set $X_{1}-X$ has infinitely many points. If the tangent spaces to the Teichmüller spaces of $X$ and $Y$ at their basepoints are isometric, then the Riemann surfaces $X$ and $Y$ are conformal.

Proof. By the adjointness theorem from [EG], for every invertible linear isometry $T$ of $A(X)^{*}$ onto $A(Y)^{*}$ there is an invertible linear isometry $S$ of $A(Y)$ onto $A(X)$ such that $S^{*}=T$. By the isometry theorem $S=C \alpha^{*}$, where $\alpha$ is a conformal homeomorphism of $X$ onto $Y$.

Theorem 4. If the Riemann surfaces $X$ and $Y$ are subsets of compact Riemann surfaces $X_{1}$ and $Y_{1}$, respectively, and the set $X_{1}-X$ has infinitely many points, then every holomorphic isomorphism $F$ from Teich $(X)$ onto Teich $(Y)$ is geometric.

Proof. Without loss of generality, we can assume that $F([i d])=[i d]$. Since $F$ is a Kobayashi isometry, its derivative $F^{\prime}([i d])$ is an isometry of the tangent space $A(X)^{*}$ onto $A(Y)^{*}$. By Theorem 3, there is a conformal map $g$ from $X$ onto $Y$. Since $X$ has the isometry property, Theorem 1 of [EG] says that the holomorphic automorphism $F^{-1} \circ \rho_{g}$ of $\operatorname{Teich}(X)$ is geometric. Therefore, so is $F$.

Definition 5. We call Teich $(X)$ (conformally) equivalent to $\operatorname{Teich}(Y)$ if there exists a biholomorphic mapping from Teich $(X)$ onto Teich $(Y)$.

Theorem 5. There is an uncountable family of plane domains $X_{k}$ such that the Teichmüller spaces Teich $\left(X_{k}\right)$ and Teich $\left(X_{j}\right)$ are inequivalent when $k \neq j$.

Proof. Suppose that $a_{i, 1}=i$ and $a_{i, s+1}=2^{a_{i, s}}$, and let

$$
X_{k}=\left\{0, \frac{1}{a_{k, 1}}, \frac{1}{a_{k, 2}}, \frac{1}{a_{k, 3}}, \ldots\right\}^{c} .
$$


Suppose that Teich $\left(X_{k}\right)$ is equivalent to Teich $\left(X_{j}\right)$ for two real numbers $k$ and $j$ with $k>j>1$. Then by Theorem 4, there exists a quasiconformal mapping $g$ from $X_{k}$ to $X_{j}$. By the extension property of quasiconformal mappings we can extend $g$ to a quasiconformal homeomorphism $h$ of the plane with $h(0)=0$.

Let $h\left(1 / a_{k, n}\right)=1 / a_{j, f(n)}$.

In the closed unit disk $h$ is Hölder continous with some constant $C$ and exponent $\alpha$. Therefore

$$
\begin{gathered}
\frac{1}{a_{j, f(n)}} \leq C\left(\frac{1}{a_{k, n}}\right)^{\alpha}, \\
a_{j, f(n)} \geq \frac{1}{C}\left(a_{k, n}\right)^{\alpha}, \\
\log a_{j, f(n)} \geq \alpha \log a_{k, n}-\log C, \\
\log a_{j, f(n)} \geq \frac{\alpha}{2} \log a_{k, n} \quad \text { for large } n, \\
a_{j, f(n)-1} \log 2 \geq \frac{\alpha}{2} a_{k, n-1} \log 2 \quad \text { for large } n, \\
a_{j, f(n)-1} \geq \frac{\alpha}{2} a_{k, n-1} \quad \text { for large } n .
\end{gathered}
$$

Consider the function $l(x)=2^{2^{x}}-2^{x+1}$. For $x>1$ we have

$l^{\prime}(x)=2^{2^{x}} 2^{x}(\log 2)^{2}-2^{x+1} \log 2=2^{x} \log 2\left[2^{2^{x}} \log 2-2\right]>2 \log 2[4 \log 2-2]>0$.

Therefore $l$ is increasing on $(1, \infty)$. Hence, for every $n$,

$$
\begin{gathered}
2^{2^{a_{k, n}}}-2^{a_{k, n}+1} \geq 2^{2^{a_{j, n}}}-2^{a_{j, n}+1}, \\
a_{k, n+2}-2 a_{k, n+1} \geq a_{j, n+2}-2 a_{j, n+1}, \\
a_{k, n+2}-a_{j, n+2} \geq 2\left(a_{k, n+1}-a_{j, n+1}\right) .
\end{gathered}
$$

Therefore, $a_{k, n}-a_{j, n} \rightarrow \infty$ as $n \rightarrow \infty$, and that implies $\frac{a_{k, n}}{a_{j, n}}=2^{a_{k, n-1}-a_{j, n-1}} \rightarrow$ $\infty$ as $n \rightarrow \infty$. Therefore,

$$
\frac{\alpha}{2} a_{k, n-1}>a_{j, n-1} \quad \text { for large } n
$$

(3) and (4) imply that there exists an integer $N$ such that $f(n)>n$ when $n \geq N$, but then

$$
\left\{\frac{1}{a_{j, 1}}, \frac{1}{a_{j, 2}}, \ldots, \frac{1}{a_{j, N}}\right\} \subset\left\{h\left(\frac{1}{a_{k, 1}}\right), h\left(\frac{1}{a_{k, 2}}\right), \ldots, h\left(\frac{1}{a_{k, N-1}}\right)\right\},
$$

which is a contradiction.

\section{REFERENCES}

[A] L. V. Ahlfors, Finitely generated Kleinian groups, Amer. J. Math. 86 (1964), 413-429; 87(1965), 759. MR 29:4890; MR 31:4906

[B] L. Bers, An approximation theorem, J. Anal. Math. 14 (1965), 1-4. MR 31:2545

[EG] C. J. Earle and F.P. Gardiner, Geometric isomorphisms between infinite dimensional Teichmüller spaces, Trans. Am. Math. Soc. 348 (1996), 1163-1190. MR 96h:32034

[EK] C. J. Earle and I. Kra, On holomorphic mappings between Teichmüller spaces, Contributions to Analysis, Academic Press, New York (1974), 107-124. MR 55:3324

[F] O. Forster, Lectures on Riemann Surfaces, Springer-Verlag, New York, Heidelberg, Berlin, 1981. MR 83d:30046

[FK] H. M. Farkas and I.Kra, Riemann Surfaces, 2nd ed., Springer-Verlag, New York, Heidelberg, Berlin, 1992. MR 93a:30047

[G1] F. P. Gardiner, Approximation of infinite dimensional Teichmüller spaces, Trans. Amer. Math. Soc. 282 (1984), 367-383. MR 85f:30082 
[G2] F. P. Gardiner, Teichmüller Theory and Quadratic Differentials, Wiley-Interscience, New York, 1987. MR 88m:32044

[H] J. Hubbard, Sur les sections analytiques de la courbe universelle de Teichmüller, Mem. Am. Math. Soc. No. 166 (1976), p. 116. MR 55:3326

[K] I. Kra, Automorphic Forms and Kleinian Groups, Benjamin, Reading, Massachusetts, 1972. MR 50:10242

[R] H. Royden, Automorphisms and isometries of Teichmüller space, Advances in the Theory of Riemann Surfaces, Ann. Math. Stud. 66, Princeton University Press, 1971, 369-384. MR 44:5452

Department of Mathematics, Cornell University, IthacA, New York 14853

E-mail address: Nikola@math.cornell.edu 